\title{
How conducting polymer electrodes operate
}

Magnus Berggren and George G. Malliaras

The self-archived postprint version of this journal article is available at Linköping University Institutional Repository (DiVA):

http://urn.kb.se/resolve?urn=urn:nbn:se:liu:diva-15720.5

N.B.: When citing this work, cite the original publication.

Berggren, M., Malliaras, G. G., (2019), How conducting polymer electrodes operate, Science, 364(6437), 233-234. https://doi.org/10.1126/science.aaw9295

Original publication available at:

https://doi.org/10.1126/science.aaw9295

Copyright: American Association for the Advancement of Science http://www.aaas.org/ 


\title{
Are Conducting Polymer Electrodes Capacitive or Faradaic?
}

\author{
Magnus Berggren and George G. Malliaras
}

Organic electrochemical (OEC) devices, which use conjugated polymers in contact with an electrolyte, are attracting an increasing attention for applications in bioelectronics, energy storage, electrocatalysis and sensors ${ }^{12}$. Their operation relies on the oxidation (electron loss) or reduction (electron gain) of the polymer, which is traditionally described to include Faradaic processes ${ }^{3}$. However, recent evidence from a variety of devices based on poly(3,4-ethylenedioxythiophene) doped with poly(styrene sulfonate) (PEDOT:PSS) is consistent with a purely capacitive process ${ }^{4}$. Is PEDOT:PSS an exception or the rule? To answer this question, we need a strategy that clarifies which processes are capacitive and which are Faradaic as conjugated polymers are electrochemically doped. To do this, we propose to use solid-state physics methodology developed to understand the operation of e.g. organic light emitting diodes (OLEDs) ${ }^{5}$. This methodology can help us to clarify the mechanisms of OEC device operation and pave the way for device optimization.

At a metal/electrolyte interface, the capacitive process stores charge at a double layer, while a Faradaic process transfers charge across the interface (Fig. 1a). The former is characterised by a boxshaped cyclic voltammogram (CV) and yields a transient charging current, while the latter gives distinct peaks in the $\mathrm{CV}$ and supports a steady-state current (given a supply of redox solutes). When a conjugated polymer film is coated on the metal electrode, the situation becomes more complex! Usually a mixed CV response is observed, interpreted as a combination of Faradaic and capacitive processes involving the polymer ${ }^{6}$. CV, however, measures the outcome of a chain of events associated with mixed ionic/electronic conduction that may include semiconductor-conductor transitions, morphology changes, delocalized states and multiple charge-compensation (doping) mechanisms by e.g. oxygen. As a result, $\mathrm{CV}$ is of limited diagnostic use to understand the mechanism of operation. Instead, we suggest focussing on the elementary steps associated with the oxidation or reduction of a conjugated polymer film, and their coupling to materials properties.

This endeavour is reminiscent of that undergone by the organic electronics community to understand the physics of OLEDs, which are currently widely used in smart phone displays and TVs. ${ }^{7}$ The simplest OLED consists of an organic semiconductor sandwiched between two metal electrodes. Its operation involves (1) injection of holes and electrons into the delocalized highest occupied (HOMO) and the lowest unoccupied (LUMO) molecular orbitals of the organic layer, respectively; (2) charge transport; and (3) recombination (Fig. 1b). Experiments and modelling of archetypal device architectures that isolate and probe one elementary step at a time were of primordial importance to understanding device operation and improving OLED efficiency. For example, electrical and photoelectron spectroscopy measurements on OLEDs with different metal electrodes, showed that charge injection improves as the energy barrier at the contact decreases ${ }^{8}$. At the same time, modelling was used to understand the role of these elementary steps on parameters such as spatial distribution of electric field and charge inside the device and has supported OLED optimization ${ }^{9}$.

Translating this language to OEC devices is surprisingly straightforward and yields a clear picture of which processes would be considered capacitive or Faradaic. For electrochemical oxidation of a conjugated polymer film, for example, the elementary steps are then (1) hole injection from the metal electrode into the HOMO of the polymer, (2) hole transport within the delocalized HOMO of the polymer, (3) anion injection from the electrolyte into the polymer, (4) anion transport in the free volume between the polymer chains, and finally (5) electrostatic compensation of the two charges in the bulk of the film, see Fig. 1c. As in OLEDs, the injection and transport of the two charges are correlated through changes in local electric field and band structure. When these elementary steps 
work efficiently, the outcome will be volumetric charging of the film, which is a pure capacitive process akin to charging a stack of capacitor plates ${ }^{4}$. It is important to note that peaks in the CV may still appear due to e.g. the presence of an injection barrier or a voltage-dependent mobility ${ }^{10}$, however, the overall process remains capacitive. Faradaic processes, on the other hand, are the result of departure from this ideal picture due to poor efficiency of an elementary step or due to competing phenomena that happen in parallel: Injection of electrons at energy levels of the polymer that lie above the LUMO of molecular oxygen, for example, leads to electron transfer from the polymer to oxygen and the formation of superoxide ${ }^{11}$. Poor injection of holes or ions, or slow transport of one of the charges, will cause large interfacial fields that may trigger electrolysis and other Faradaic reactions. Finally, electron transfer between the injected ion and the polymer chain (the equivalent of recombination in an OLED) would also be a Faradaic process. Therefore, the answer to the question posed on the title will depend on materials and operating conditions. PEDOT:PSS shows capacitive behaviour because it exhibits a high mixed ionic/electronic conductivity and is stable in common electrolytes under small doping changes. Moreover, it would be interesting to extend this methodology to so-called redox polymers ${ }^{12}$ by accounting for much lower electronic carrier mobilities and highly localized states among the pendant redox groups.

Based on this understanding, experiments that isolate and probe one elementary step at a time are suggested as the next stage in advancing the field of OEC devices. Transfer of knowledge from the field of organic electronics, where our understanding of injection and transport of electronic charges into polymers is well developed, requires accounting for the impact of electrolytes on film morphology and dielectric environment. Techniques such as "moving front" measurements, chronoamperometry, and the recording of organic electrochemical transistor parameters can help advance our understanding of ion injection and transport and its coupling to electronic conductivity and energetics, at different doping densities. Finally, an energy level description of OEC devices will support the development of more sophisticated devices and will also help us to understand the relationship between polymer energetics, charge transport and reactivity. With this understanding at hand, Faradaic reactions can be promoted or supressed by tuning polymer energetics and the spatial distribution of electric field and charge: In neural electrodes, where capacitive behaviour is desirable to avoid the generation of reactive species, care should be taken to avoid interfacial fields. Conversely, in electrocatalysis, engineering electron transfer to solutes throughout the volume of the polymer film will help achieve a large steady-state current and maximize efficiency. 
Fig. 1

Capacitive process

a)
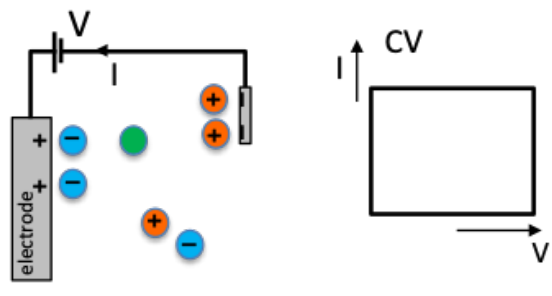

Faradaic process
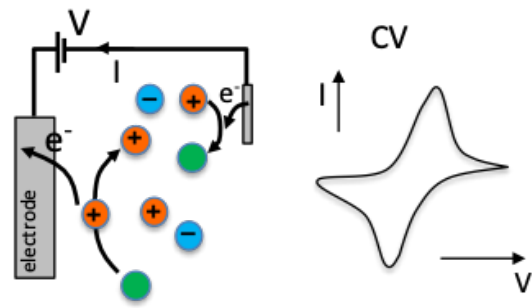

b)
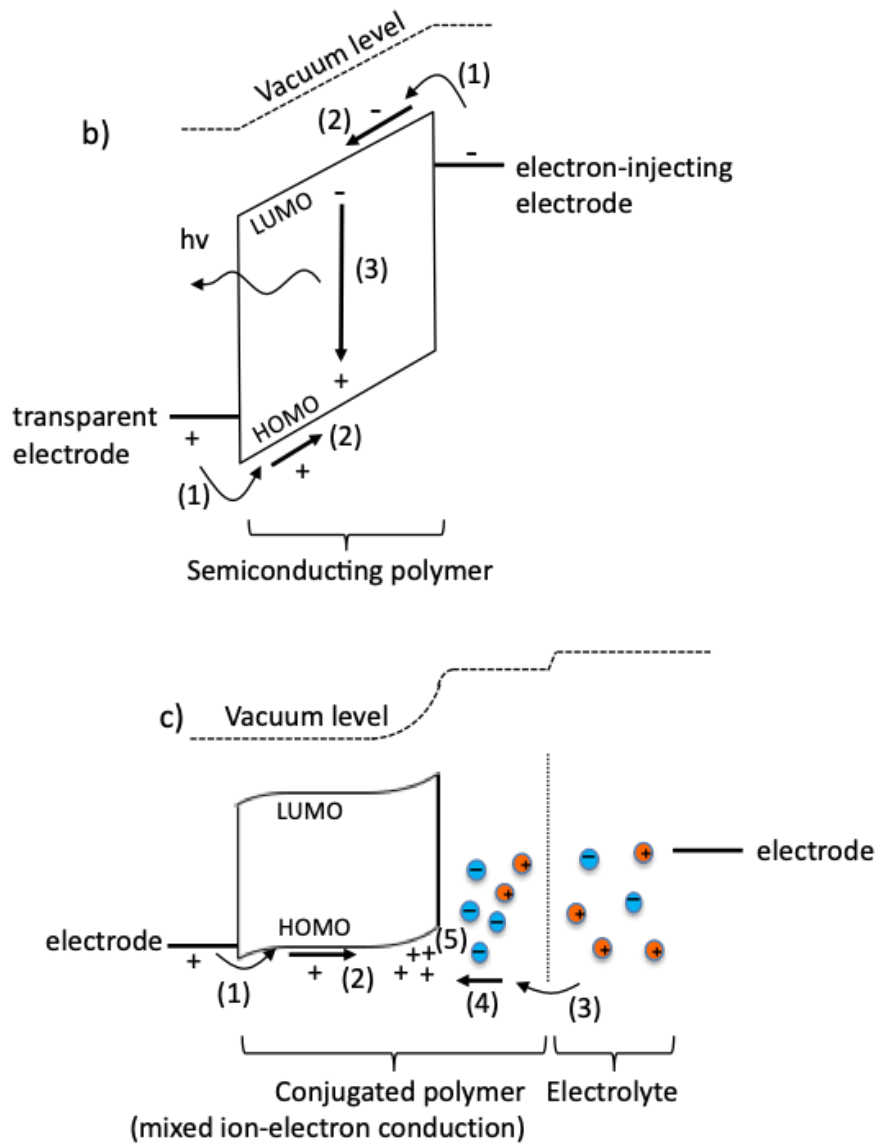

Figure 1: (a) Capacitive vs Faradaic processes at metal/electrolyte interfaces. The former leads to formation of a double layer capacitor between electronic and ionic charges, while the latter involves electron transfer to redox active solutes in the electrolyte. Conjugated polymers (b) OLED energy level diagram showing the Fermi levels of the two metal electrodes and the HOMO and LUMO of the organic film, as well as the elementary steps of hole and electric injection, transport and recombination. (c) Simplified energy level diagram for electrochemical oxidation of a conjugated polymer showing the elementary steps involved in the process.

1. Song, Z.; Zhou, H., Towards sustainable and versatile energy storage devices: An overview of organic electrode materials. Energy and Environmental Science 2013, 6 (8), 2280-2301.

2. Rivnay, J.; Inal, S.; Salleo, A.; Owens, R. M.; Berggren, M.; Malliaras, G. G., Organic electrochemical transistors. Nature Reviews Materials 2018, 3.

3. Feldberg, S. W., Reinterpretation of Polypyrrole Electrochemistry. Consideration of Capacitive Currents in Redox Switching of Conducting Polymers. Journal of the American Chemical Society 1984, 106 (17), 4671-4674.

4. Proctor, C. M.; Rivnay, J.; Malliaras, G. G., Understanding volumetric capacitance in conducting polymers. Journal of Polymer Science, Part B: Polymer Physics 2016, 54 (15), 1433-1436.

5. Friend, R. H.; Gymer, R. W.; Holmes, A. B.; Burroughes, J. H.; Marks, R. N.; Taliani, C.; Bradley, D. D. C.; Dos Santos, D. A.; Brédas, J. L.; Lögdlund, M.; Salaneck, W. R., Electroluminescence in conjugated polymers. Nature 1999, 397 (6715), 121-128.

6. Heinze, J.; Frontana-Uribe, B. A.; Ludwigs, S., Electrochemistry of conducting polymerspersistent models and new concepts. Chemical Reviews 2010, 110 (8), 4724-4771. 
7. Sirringhaus, H.; Tessler, N.; Friend, R. H., Integrated optoelectronic devices based on conjugated polymers. Science 1998, 280 (5370), 1741-1744.

8. Koch, N., Organic electronic devices and their functional interfaces. ChemPhysChem 2007, 8 (10), 1438-1455.

9. $\quad$ Arkhipov, V. I.; Emelianova, E. V.; Tak, Y. H.; Bässler, H., Charge injection into light-emitting diodes: Theory and experiment. Journal of Applied Physics 1998, 84 (2), 848-856.

10. Tybrandt, K.; Zozoulenko, I. V.; Berggren, M., Chemical potential-electric double layer coupling in conjugated polymer-polyelectrolyte blends. Science Advances 2017, 3 (12).

11. Miglbauer, E.; Wójcik, P. J.; Głowacki, E. D., Single-compartment hydrogen peroxide fuel cells with poly(3,4-ethylenedioxythiophene) cathodes. Chemical Communications 2018, 54 (84), 1187311876.

12. Choi, W.; Harada, D.; Oyaizu, K.; Nishide, H., Aqueous electrochemistry of poly(vinylanthraquinone) for anode-active materials in high-density and rechargeable polymer/air batteries. Journal of the American Chemical Society 2011, 133 (49), 19839-19843. 